\title{
Electronic and magnetic properties of graphane nanoribbons
}

\author{
H. Şahin, ${ }^{1}$ C. Ataca, ${ }^{1,2}$ and S. Ciraci ${ }^{1,2, *}$ \\ ${ }^{1}$ UNAM-Institute of Materials Science and Nanotechnology, Bilkent University, 06800 Ankara, Turkey \\ ${ }^{2}$ Department of Physics, Bilkent University, 06800 Ankara, Turkey \\ (Received 27 January 2010; revised manuscript received 26 April 2010; published 12 May 2010)
}

\begin{abstract}
In this study, we investigate the electronic and magnetic properties of graphane nanoribbons. We find that zigzag and armchair graphane nanoribbons with H-passivated edges are nonmagnetic semiconductors. While bare armchair nanoribbons are also nonmagnetic, adjacent dangling bonds of bare zigzag nanoribbons have antiferromagnetic ordering at the same edge. Band gaps of the H-passivated zigzag and armchair nanoribbons exponentially depend on their width. Detailed analysis of adsorption of $\mathrm{C}, \mathrm{O}, \mathrm{Si}, \mathrm{Ti}, \mathrm{V}, \mathrm{Fe}, \mathrm{Ge}$, and Pt atoms on the graphane ribbon surface reveal that functionalization of graphane nanoribbons is possible via these adatoms. It is found that $\mathrm{C}, \mathrm{O}, \mathrm{V}$, and $\mathrm{Pt}$ atoms have tendency to replace $\mathrm{H}$ atoms of graphane. We showed that significant spin polarizations in graphane can be achieved through creation of domains of $\mathrm{H}$ vacancies and $\mathrm{CH}$ divacancies.
\end{abstract}

DOI: 10.1103/PhysRevB.81.205417

PACS number(s): 73.22.Pr, 75.75.-c, 61.48.-c, 62.23.Kn

\section{INTRODUCTION}

After the synthesis of two-dimensional (2D) graphene, ${ }^{1-6}$ its nanoribbons (NRs) have been a subject of interest, both experimentally ${ }^{7,8}$ and theoretically. ${ }^{9-15}$ Advances in experimental techniques are paving the way for integrating the exceptional electrical, optical, and magnetic functionalities of these nanometer-sized materials into future electronic technology. In addition to numerous theoretical and experimental works on graphene and its NRs, research efforts have been also devoted to synthesize its various derivatives. Notably, the synthesis of a $2 \mathrm{D}$ hydrocarbon in honeycomb structure, ${ }^{16}$ namely, graphane, followed its prediction through theoretical works. ${ }^{17,18}$ Detailed analysis of hydrogenation processes of graphene leading to graphane and the existence of hydrogen (H) frustrations were discussed very recently. ${ }^{19}$ Furthermore, it was reported that graphane NRs have more favorable formation energies than experimentally available graphene ribbons..$^{20}$ Recently, we reported the possibility of obtaining magnetization through dehydrogenation of domains on $2 \mathrm{D}$ graphane. ${ }^{21}$ Modification of electronic structure of graphane by introducing either a hydroxyl group or a $\mathrm{H}$ vacancy was also investigated with GW self-energy calculations.22 $\mathrm{Al}$ though there are several recent studies on graphane, electrical and magnetic properties of its NRs have remained unexplored.

Recent advances in graphane have motivated us to explore the properties of zigzag and armchair graphane NRs. In this paper, using first-principles plane-wave method within the density-functional theory (DFT) we investigated the electronic and magnetic properties of bare and $\mathrm{H}$-passivated graphane NRs. We also explored the effects of specific imperfections on these properties. These are various foreign atoms adsorbed on the surfaces of graphane NRs, vacancies, and edge roughness. We found that these imperfections can attribute interesting functionalities by modifying the electronic and magnetic properties of graphane NRs.

\section{METHODS}

We perform first-principles calculations ${ }^{23}$ within DFT using projector augmented wave potentials ${ }^{24}$ and approximate exchange-correlation functional by spin-polarized localdensity approximation ${ }^{25}$ (LDA). Kinetic energy cutoff $\hbar^{2} \mid \mathbf{k}$ $+\left.\mathbf{G}\right|^{2} / 2 m$ for plane-wave basis set is taken as $500 \mathrm{eV}$. In the self-consistent potential and total-energy calculations of $2 \mathrm{D}$ graphane, a set of $(35 \times 35 \times 1) \mathbf{k}$-point sampling is used for Brillouin-zone (BZ) integration. The convergence criterion of self-consistent calculations for ionic relaxations is $10^{-5} \mathrm{eV}$ between two consecutive steps. By using the conjugate-gradient method, all atomic positions and unit cells are optimized until the atomic forces are less than $0.03 \mathrm{eV} / \AA$. Pressures on the lattice unit cell are decreased to values less than $1.0 \mathrm{kbar}$.

In order to correct the energy bands and band-gap values obtained by LDA, frequency-dependent $\mathrm{GW}_{0}$ calculations are carried out. ${ }^{26}$ Screened Coulomb potential $W$, is kept fixed at initial DFT value $\mathrm{W}_{0}$ and Green's function $G$, is iterated five times. $\mathrm{GW}_{0}$ self-energy calculations are carried out with $15 \AA$ vacuum spacing, default cutoff potential, 160 bands, and 64 grid points.

Graphane NRs are treated by the supercell geometry within periodic boundary conditions. Vacuum spacing of at least $15 \AA$ is placed between adjacent graphane NRs to hinder the interactions. In specific cases double unit cell is used in our calculations to allow the possible antiferromagnetic (AFM) orderings along the ribbon edges. Reciprocal space integrations are carried out with $1 \times 1 \times 15$ MonkhorstPack k-point grids.

\section{TWO-DIMENSIONAL GRAPHANE}

For a better understanding of graphane NRs we start with 2D graphane which is derived from the hydrogenation of graphene, where each carbon atom is saturated by a single hydrogen atom. Accordingly, the primitive cell of graphane consists of two carbon and two hydrogen atoms. Chairlike configuration of infinite sheet of $2 \mathrm{D}$ graphane is formed, whereby each carbon atoms of A- and B-type sublattices are saturated by hydrogen atoms from above and below, respectively, as described in Fig. 1(a). This is known as the most favorable and stable hydrocarbon conformation. ${ }^{17,21}$ The pla- 

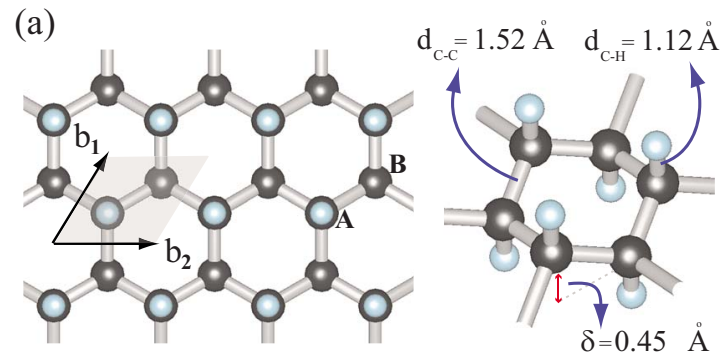

(b)
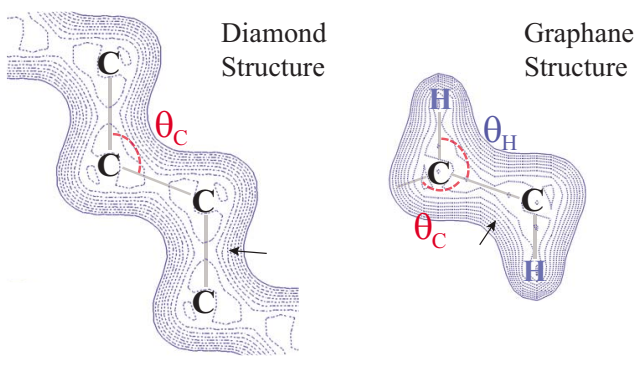

(c)

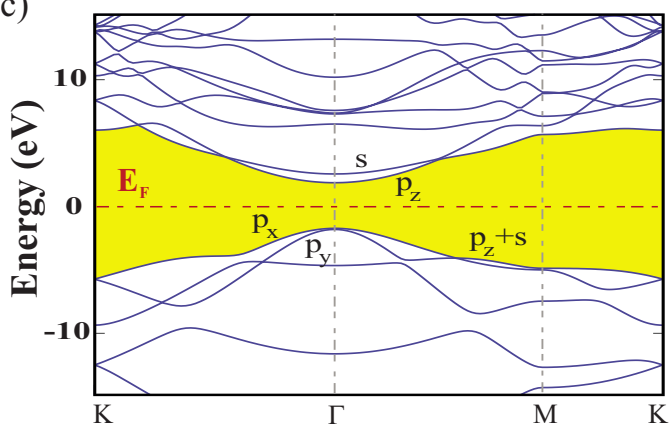

FIG. 1. (Color online) (a) Top and perspective view of the atomic structure of infinite 2D graphane sheet having honeycomb structure. Two sublattices of graphane are indicated by A and B. Black (dark) and blue (light) balls are for carbon and hydrogen atoms, respectively. (b) Charge density contour plots of diamond and graphane are shown on a plane passing through $\mathrm{C}-\mathrm{C}-\mathrm{C}-\mathrm{C}$ and $\mathrm{H}-\mathrm{C}-\mathrm{C}-\mathrm{H}$ bonds, respectively. The tetrahedral angle of the diamond $\theta_{\mathrm{C}}=109.47^{\circ}$. Arrows indicate the direction of increasing charge density. The calculated values of $\theta_{\mathrm{C}}$ and $\theta_{\mathrm{H}}$, namely, C-C-C and $\mathrm{H}-\mathrm{C}-\mathrm{C}$ bond angles in graphane, respectively, are given in Table I. Contour spacings are $0.0286 \mathrm{e} / \AA^{3}$. (c) The LDA energy-band structure where the orbital character of specific bands is also given. The band gap is shaded yellow/gray.

nar graphene honeycomb structure, which is stabilized by planar $s p^{2}$-hybrid orbitals and $\pi$ bonding between adjacent perpendicular $p_{z}$ orbitals is puckered (buckled) as a result of the adsorption of $\mathrm{H}$ atoms, whereby a single $\mathrm{C}$ atom is bound to three adjacent $\mathrm{C}$ atoms and a single $\mathrm{H}$ atom through "tetrahedrally coordinated" $s p^{3}$-like hybrid orbitals. The buckling, $\delta$, i.e., the perpendicular distance between A-type and B-type carbon sublattices, is calculated to be $0.45 \AA$. As a result, the lattice constant of graphene increases from 2.46 to $2.51 \AA$ and hence the $\mathrm{C}-\mathrm{C}$ and $\mathrm{C}-\mathrm{H}$ bond lengths become $1.52 \AA$ and $1.12 \AA$, respectively, as indicated in Fig. 1(a). The charge-density contour plots given in Fig. 1(b) indicate that the charge distribution in graphane especially around the $\mathrm{C}-\mathrm{C}$ bond is reminiscent of that in tetrahedrally coordinated diamond. In graphane, the angle $\theta_{\mathrm{H}}$ between $\mathrm{H}-\mathrm{C}$ and $\mathrm{C}-\mathrm{C}$ bonds and $\theta_{\mathrm{C}}$ between adjacent $\mathrm{C}-\mathrm{C}$ bonds are $107.35^{\circ}$ and $111.51^{\circ}$, respectively. The mean value of these angles is equal to the tetrahedral angle $\theta_{\mathrm{C}}$ of diamond. The maximum of the $\mathrm{C}-\mathrm{C}$ bond charge in diamond and in graphane are found to be $\sim 0.282 \mathrm{e} / \AA^{3}$ and $\sim 0.286 \mathrm{e} / \AA^{3}$, respectively.

The cohesive energy of graphane (per unit cell) relative to free $\mathrm{C}$ and $\mathrm{H}$ atoms is obtained from $E_{\mathrm{c}}=2 \times E_{T}^{\mathrm{H}}+2 \times E_{T}^{\mathrm{C}}$ $-E_{T}^{G r a}$, where $E_{T}^{\mathrm{H}}$ and $E_{T}^{\mathrm{C}}$ are the total energies of single free $\mathrm{C}$ and free $\mathrm{H}$, whereas $E_{T}^{G r a}$ is the total energy of graphane. The cohesive energy depends whether the energies of magnetic or nonmagnetic (NM) states of free $\mathrm{C}$ and free $\mathrm{H}$ atoms are considered. Here the cohesive energy per unit cell of graphane is calculated to be $23.57(27.65) \mathrm{eV}$ by considering the magnetic (nonmagnetic) states of free atoms. As for the average $\mathrm{C}-\mathrm{H}$ bond energy, one can use the formula $E_{\mathrm{C}-\mathrm{H}}=$ $\left(-E_{T}^{G r a}+E_{T}^{G r e}+2 \times E_{T}^{\mathrm{H}}\right) / 2$, where $E_{T}^{G r e}$ is the total energy of graphene. The calculated value is 2.84 (3.74 for nonmagnetic case). We reported ${ }^{21}$ that the desorption of a single $\mathrm{H}$ atom from the graphane is an endothermic reaction with an energy of $4.79 \mathrm{eV}$. When compared with the energy of $\mathrm{C}-\mathrm{H}$ bond, the desorption energy of single $\mathrm{H}$ is larger since the single $\mathrm{H}$ removal creates reconstruction of nearby atoms which further reduces the total energy of the system.

Due to $s p^{3}$ saturation of $\mathrm{C}$ atoms, $2 \mathrm{D}$ graphane is a NM semiconductor with a direct band gap of $3.42 \mathrm{eV}$ as shown in Fig. 1(c). However, this band gap, which is underestimated within DFT, is corrected by $\mathrm{GW}_{0}$ approximation to become $5.97 \mathrm{eV}^{21,22}$ While the top of the valence band originates from $p_{x}$ and $p_{y}$ orbitals of $\mathrm{C}$ atoms, the bottom of the conduction band has $\mathrm{C} p_{z}$ orbital character. Earlier, we also calculate $^{21}$ the phonon modes of infinite $2 \mathrm{D}$ graphane, which yield all real frequencies in BZ. Having all frequencies positive indicate the stability of graphane structure.

An exceptional feature of graphane is related with its interesting charging configuration. While graphene has a covalent bonding, upon the saturation of each $\mathrm{C}$ atom by a single $\mathrm{H}$ atom, a charge of $\delta Q \sim 0.1$ electrons is transferred from $\mathrm{H}$ to $\mathrm{C}$. At the end, the negatively charged bilayer of carbon atoms becomes sandwiched between positively charged $\mathrm{H}$ layers. Graphane having this charging has the photoelectric threshold (work function) calculated to be $\Phi=4.97 \mathrm{eV}$, which is $0.2 \mathrm{eV}$ larger than that of graphene. In Table I, we presented the calculated values related with structure, energy bands, photoelectric threshold, etc., of graphane and graphene for the sake of comparison. To be complete Table I also includes calculated elastic constants such as in-plane stiffness ${ }^{27} C\left[=\frac{1}{A}\left(\frac{\partial^{2} E_{T}}{\partial \epsilon^{2}}\right)\right.$, where $E_{T}$ is the total energy of the system under strain, $\epsilon$ is the uniaxial strain, and $A$ is the area of the unit cell] and Poisson's ratio ${ }^{28} \nu$.

\section{GRAPHANE NANORIBBONS}

The nanoribbons cut from a 2D graphane are structures providing important features for various technological applications. Two major families of graphane NRs are distinguished depending on their orientations, namely, armchair and zigzag graphane NRs. Apart from the orientation, graphane NRs are characterized by their widths. For armchair graphane NRs, the width, $N$, is defined by the number of C-C dimers in the unit cell which are parallel to the axis of 
TABLE I. Comparison of the calculated quantities of graphene and graphane. Lattice constant, $a$; C-C bond distance, $d_{\mathrm{C}-\mathrm{C}}$; C-H bond distance, $d_{\mathrm{C}-\mathrm{H}}$; the buckling, $\delta$ [see Fig. 1(a)]; angle between adjacent $\mathrm{C}-\mathrm{C}$ bonds, $\theta_{\mathrm{C}}$ [see Fig. 1(b)]; angle between adjacent $\mathrm{C}-\mathrm{H}$ and $\mathrm{C}-\mathrm{C}$ bonds, $\theta_{\mathrm{H}}$; energy band gap calculated by LDA, $E_{g}^{\mathrm{LDA}}$; energy band gap corrected by $\mathrm{GW}_{0}, E_{g}^{\mathrm{GW}_{0}}$; cohesive energy $E_{\mathrm{c}}^{\mathrm{NM}},\left(E_{\mathrm{c}}^{m}\right)$ obtained with respect to nonmagnetic (magnetic) free-atom energies; the $\mathrm{C}-\mathrm{H}$ bond energy, $E_{\mathrm{C}-\mathrm{H}}^{\mathrm{NM}}\left(E_{\mathrm{C}-\mathrm{H}}^{m}\right)$ obtained with respect to nonmagnetic (magnetic) free-atom energies; photoelectric threshold (work function), $\Phi$; in-plane stiffness, $C$ and Poison ratio, $\nu$.

\begin{tabular}{lccccccccccccccc}
\hline \hline Material $(1 \times 1)$ unit cell & $\begin{array}{c}a \\
(\AA)\end{array}$ & $\begin{array}{c}d_{\mathrm{C}-\mathrm{C}} \\
(\AA)\end{array}$ & $\begin{array}{c}d_{\mathrm{C}-\mathrm{H}} \\
(\AA)\end{array}$ & $\begin{array}{c}\delta \\
(\AA)\end{array}$ & $\begin{array}{c}\theta_{\mathrm{C}} \\
(\mathrm{deg})\end{array}$ & $\begin{array}{c}\theta_{\mathrm{H}} \\
(\mathrm{deg})\end{array}$ & $\begin{array}{c}E_{g}^{\mathrm{LDA}} \\
(\mathrm{eV})\end{array}$ & $\begin{array}{c}E_{g}^{\mathrm{GW}} \\
(\mathrm{eV})\end{array}$ & $\begin{array}{c}E_{\mathrm{c}}^{\mathrm{NM}} \\
(\mathrm{eV})\end{array}$ & $\begin{array}{c}E_{\mathrm{c}}^{m} \\
(\mathrm{eV})\end{array}$ & $\begin{array}{c}E_{\mathrm{C}-\mathrm{H}}^{\mathrm{NM}} \\
(\mathrm{eV})\end{array}$ & $\begin{array}{c}E_{\mathrm{C}-\mathrm{H}}^{m} \\
(\mathrm{eV})\end{array}$ & $\begin{array}{c}\Phi \\
(\mathrm{eV})\end{array}$ & $\begin{array}{c}C \\
\left.(\mathrm{~J} / \mathrm{m})^{2}\right)\end{array}$ & $\begin{array}{c}\nu \\
\nu\end{array}$ \\
\hline Graphene & 2.46 & 1.42 & & & 120 & & 0.00 & 0.00 & 20.16 & 17.87 & & 4.77 & $335^{\mathrm{a}}$ & 0.16 \\
Graphane & 2.51 & 1.52 & 1.12 & 0.45 & 111.51 & 107.35 & 3.42 & 5.97 & 27.65 & 23.57 & 3.74 & 2.84 & 4.97 & $243^{\mathrm{b}}$ & 0.07 \\
\hline \hline
\end{tabular}

${ }^{\text {aReference } 29 .}$

${ }^{\mathrm{b}}$ Reference 30 .

the nanoribbon; for zigzag graphane NRs, $N$ denotes the number of zigzag carbon chains along the nanoribbon axis. The electronic structure changes depending on whether the dangling bonds of carbon atoms at the edge are free (bare) or passivated by single $\mathrm{H}$ atoms. In Figs. 2(a) and 2(b) we show the atomic and electronic structure of armchair and zigzag graphane NRs with $N=11$ and $N=6$, respectively.

Bare armchair graphane NR is a NM indirect band-gap semiconductor since the electrons at the dangling orbitals are paired. The band gap of $2 \mathrm{D}$ graphane is reduced due to the bands of edge states of dangling bonds occurring in the band gap. The charge-density analysis of these edge state bands presented in Fig. 2(a) clearly shows that they are localized at the edges of the NR. Upon hydrogen passivation of the threefold coordinated edge atoms, the edge state bands in the gap disappear and the direct band gap opens up.

Similar to armchair graphane NRs, the dangling bonds of the threefold coordinated carbon atoms at both edges of zigzag graphane NRs give rise to edge states in the band gap. Owing to relatively large distance between adjacent dangling bonds and hence their relatively smaller coupling, they have a minute dispersion. Charge density isosurfaces of these edge states confirm their localization at the edges. Also, owing to their weak coupling the spins of adjacent dangling bonds cannot be paired. Consequently, each carbon atom at the zigzag edge attains a magnetic moment of 1 Bohr magneton $\left(\mu_{B}\right)$. The ordering of these magnetic moments is however dramatically different from that of the zigzag graphene NRs. The significant coupling between two spin states at different edges gives rise to AFM ordering along the graphene edge.

The physical origin of the magnetic interactions between the edges of graphitic fragments was treated earlier. ${ }^{9}$ For zigzag graphene nanoribbon, collective alignment of the magnetic moments is along the same ribbon edge through the ferromagnetic (FM) interaction but AFM ordering between opposite edges is attributed to magnetic-tail interactions. The magnetic ordering of a wide zigzag graphane NRs $(\sim 2 \mathrm{~nm})$ are examined in a supercell comprising two unit cells, whereby antiferromagnetic ordering between adjacent dangling bonds at the same edge is allowed. We consider four different orderings, such as AFM-AFM (where, respectively, the ordering of magnetic moments at the atoms located at different and same edge are antiferromagnetic), FMAFM, AFM-FM, and FM-FM as described in Fig. 3. Here the difference charge density is defined as the $\Delta \rho=\rho^{(\uparrow)}$ $-\rho^{(\downarrow)}$, where $\rho^{\uparrow(\downarrow)}$ is spin-up (spin-down) charge. Since the magnetic interaction between two edges vanishes, AFMAFM and FM-AFM orderings have the same total energy corresponding to the ground state. These orderings are 125 $\mathrm{meV}$ energetically more favorable than the AFM-FM and

(a)

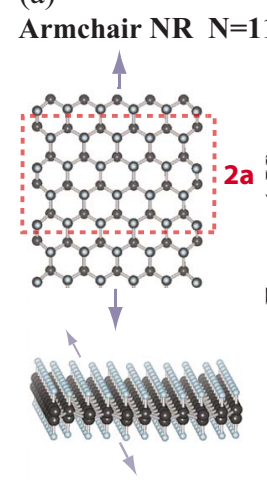

(b)
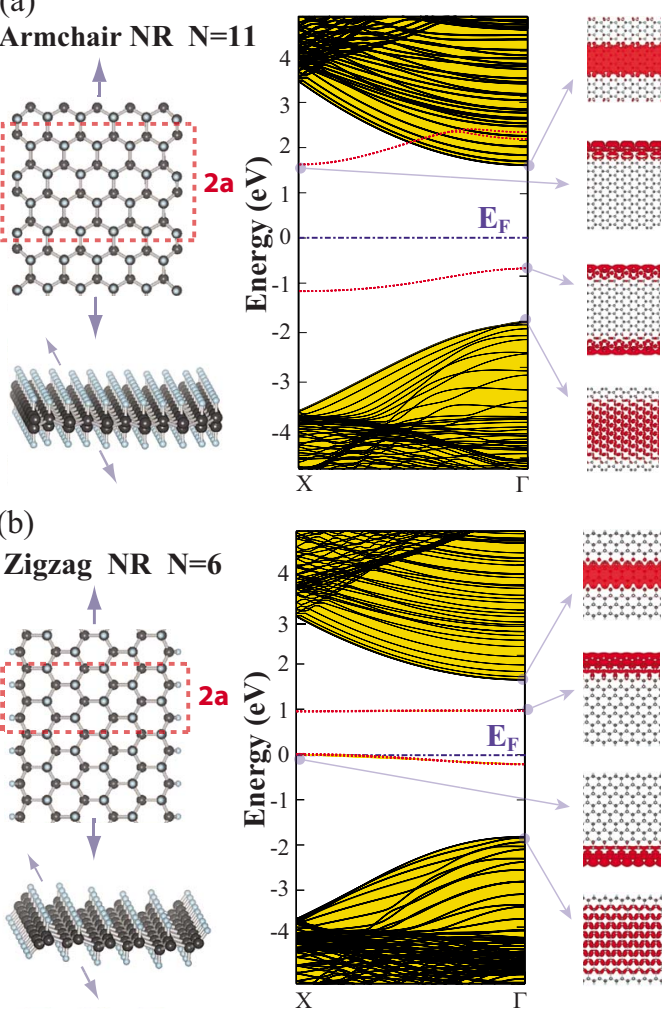

FIG. 2. (Color online) (a) Atomic structure of bare armchair graphane NR having $N=11$. The double unit cell of the nanoribbon is delineated by red/dashed lines with the lattice constant $2 a$. Large/ black and small/light blue balls indicate carbon and hydrogen atoms. Energy-band structure corresponding to the armchair graphane $\mathrm{NR}$ and charge density of selected bands are shown in the panels at the righthand side. (b) Atomic structure of bare zigzag graphane NR having $N=6$ with double unit cell delineated by red/dashed lines and with lattice constant $2 a$. Energy-band structure and isosurface charge density of selected states corresponding to zigzag graphane NR are indicated. Bands shown by red/dotted lines are derived from edge states. Zero of energy is set to the Fermi level, shown by dashed-dotted lines, of the nanoribbons with H-passivated edges. In spin polarized calculations double unit cell is used to allow antiferromagnetic order along the edges. 


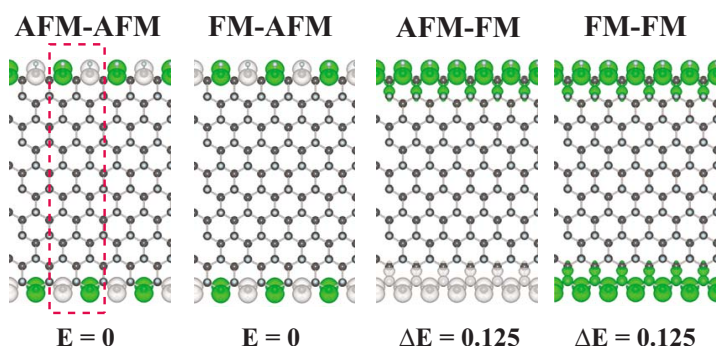

FIG. 3. (Color online) Total energies of possible magnetic orderings at the edges of bare zigzag graphane nanoribbons. Calculations are performed in double unit cell delineated by red/dashed lines. Spin-up and spin-down states are shown by green/dark and gray/light isosurfaces of the difference charge density, $\Delta \rho$.

FM-FM orderings. From this analysis it is revealed that AFM spin alignment between adjacent atoms at the same edge is preferred by the zigzag graphane NRs. FM ordering between two spins has $62.5 \mathrm{meV}$ higher energy. For zigzag graphane NR with $N=6$, the magnetic coupling between two edges is negligible and hence either AFM-AFM or FM-AFM orderings have the same energy and they are ground states. However, when the width of the nanoribbon is less than $12 \AA$ the degeneracy of AFM-AFM and FM-AFM states is broken. The bare zigzag graphane NR is an indirect and antiferromagnetic semiconductor with an indirect band gap relatively smaller than that of 2D graphane. However, it becomes NM, direct band-gap semiconductor upon passivation of the dangling bonds. Also the magnetic edge states disappear and band gap becomes larger.

The value of the band gap of graphane NRs can differ from that of parent 2D structure due to a combined effects of quantum confinement, edge state bands as well as folding in the direction perpendicular to the NR axis. We note that the band gap of graphane NR depends on its width given by $N$. However, the quantum confinement effect is not as emphasized as that found in graphene NR. In Fig. 4, H-passivated armchair and zigzag graphane NRs display a quantum confinement effect, namely, the band-gap reduction with increasing width or $N$. This behavior is fitted to an expression as

$$
E_{\text {gap }}(N)=3.42 \mathrm{eV}+\alpha \exp (-N \beta) .
$$

Here $\alpha$ and $\beta$ are fitting parameters. The values of $\alpha$ and $\beta$ are found to be 1.18 and $0.19 \mathrm{eV}$ for zigzag graphane NRs

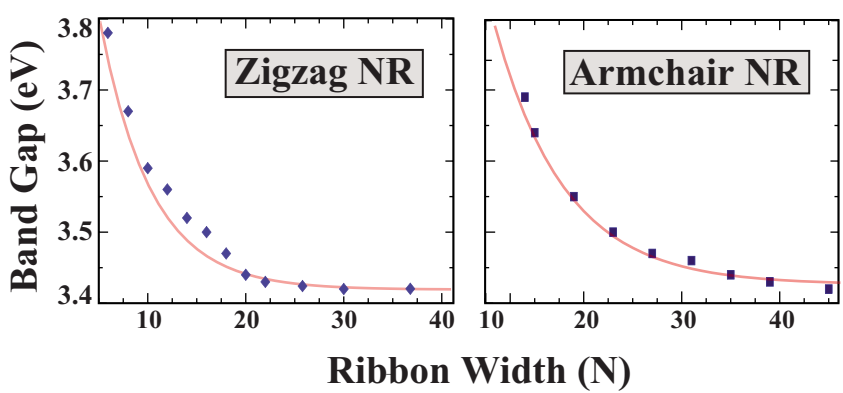

FIG. 4. (Color online) Variation in the energy band gap of $\mathrm{H}$-passivated zigzag and armchair NRs of graphane as a function of width given by $N$. The variation in the band gap with $N$ is fitted to the curve given by continuous line. (See text.)

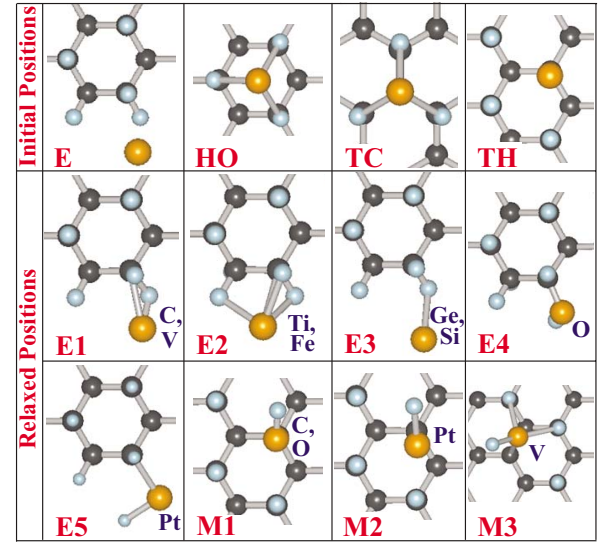

FIG. 5. (Color online) Schematic representations of possible positions of adatoms on a large H-passivated armchair graphane nanoribbon. Positions of adatoms obtained after the structure optimization through minimization of total energy and forces exerting on the atoms are also shown.

(2.15 and $0.14 \mathrm{eV}$ for armchair graphane NRs). The band gaps of both types of graphane NRs go to $3.42 \mathrm{eV}$ as $N$ $\rightarrow \infty$. Considering the $\mathrm{GW}_{0}$ corrected value of the band gap, $5.97 \mathrm{eV}$, the above fitting can be expressed as $E_{\text {gap }}(N)$ $=5.97+\alpha \exp (-N \beta)$ assuming that the same scissor operation is applicable for graphane NRs. Apparently, the bandgap values of graphane NRs calculated using DFT are underestimated and hence their values are expected to occur $\sim 2.5 \mathrm{eV}$ larger than presented in Fig. 4. Unfortunately, $\mathrm{GW}_{0}$ self-energy corrections cannot be performed for graphane NRs due to large computational time. We note that the variation in band gap is relevant for $N<22$ for zigzag graphane NRs and $N<30$ for armchair graphane NRs. In Ref. 20 the band gaps of hydrogen saturated graphane NRs were calculated for $6 \leq N \leq 15$ using generalized gradient approximation (GGA). In the present study band gaps are calculated for $10 \leq N \leq 40$ using LDA. The hydrogenated graphane NRs included in both studies, namely, those $10 \leq N \leq 15$ are in good agreement. Largest band-gap difference of $0.1 \mathrm{eV}$ found between two studies is attributed to different approximations (i.e., LDA versus GGA) in representing exchangecorrelation potential.

\section{FUNCTIONALIZATION OF GRAPHANE NRs BY ADATOMS}

Adsorption of adatoms is widely used and an efficient way to provide new functionalities to structures in nanoscale applications. ${ }^{31-33}$ Adsorption of $\mathrm{C}, \mathrm{O}, \mathrm{Si}, \mathrm{Ge}, \mathrm{Pt}, \mathrm{V}, \mathrm{Fe}$, and Ti adatoms on graphane nanoribbons is investigated by using $(1 \times 1 \times 6)$ supercell of $\mathrm{H}$-passivated armchair graphane NR having $N=8$. Adsorption geometry is determined by calculating the lowest binding energy corresponding to the optimized structures. To this end, the binding energies of adatoms at four initial sites described in Fig. 5: these are edge site $\mathrm{E}$, hollow site $\mathrm{HO}$, top site of the carbon $\mathrm{TC}$, and top site of the hydrogen atom TH in the middle of NR. The stable binding sites are determined upon structure relaxation. In the 
TABLE II. Summary of the calculated quantities for adatoms adsorbed on a H-passivated armchair graphane NR. The first and second lines in each row associated with a given adatom adsorbed to edge site and the sites near the center of the graphane NRs, respectively. $d_{\mathrm{H}}$, the adatom nearest hydrogen distance; $d_{\mathrm{C}}$, the nearest adatom carbon distance; $E_{b}$, adatom binding energy; $\mu_{T}$, magnetic moment per supercell; $\rho^{*}$, excess charge on the adatom (where negative sign indicates excess electrons); $\Phi$, photoelectric threshold (work function); $p$, dipole moment calculated in the direction normal to graphane NR surface; $E_{i}$ energies of localized states associated with adatoms. Localized states are measured from the top of the valence bands in electron volt. The occupied ones are indicated by bold numerals and their spin alignments are denoted by either $\uparrow$ or $\downarrow$. Up to first seven adatom states of $E_{i}$ are shown.

\begin{tabular}{|c|c|c|c|c|c|c|c|c|c|}
\hline Atom & Site & $\begin{array}{l}d_{\mathrm{H}} \\
(\AA)\end{array}$ & $\begin{array}{l}d_{\mathrm{C}} \\
(\AA)\end{array}$ & $\begin{array}{c}E_{b} \\
(\mathrm{eV})\end{array}$ & $\begin{array}{c}\mu_{T} \\
\left(\mu_{B}\right)\end{array}$ & $\begin{array}{l}\rho^{*} \\
(\mathrm{e})\end{array}$ & $\begin{array}{c}\Phi \\
(\mathrm{eV})\end{array}$ & $\begin{array}{c}p \\
(\mathrm{e} \times \AA)\end{array}$ & $E_{i} \uparrow:$ spin-up, $\downarrow$ : spin-down states \\
\hline \multirow[t]{2}{*}{$\mathrm{C}$} & $\mathrm{E} \rightarrow \mathrm{E} 1$ & 1.27 & 2.05 & 1.10 & 2.00 & -0.28 & 3.85 & 0.02 & $\mathbf{0 . 4 5} \uparrow, \mathbf{0 . 8 8} \uparrow, 2.17 \downarrow, 2.88 \downarrow, 3.53 \uparrow, 3.64 \downarrow$ \\
\hline & $\mathrm{TH} \rightarrow \mathrm{M} 1$ & 1.12 & 1.45 & 3.99 & 0.00 & -0.18 & 4.02 & 0.34 & $0.72,1.52$ \\
\hline \multirow[t]{2}{*}{$\mathrm{O}$} & $\mathrm{E} \rightarrow \mathrm{E} 4$ & 0.98 & 1.42 & 5.67 & 0.00 & -1.23 & 4.63 & 0.19 & $-\mathbf{0 . 8 7},-\mathbf{0 . 5 0},-\mathbf{0 . 2 2}, \mathbf{0 . 0 0}, 4.80$ \\
\hline & $\mathrm{TH} \rightarrow \mathrm{M} 1$ & 0.98 & 1.43 & 5.89 & 0.00 & -1.27 & 4.59 & 0.11 & $-\mathbf{1 . 1 6},-\mathbf{1 . 0 2},-\mathbf{0 . 6 6}, 4.08$ \\
\hline \multirow[t]{2}{*}{$\mathrm{Si}$} & $\mathrm{E} \rightarrow \mathrm{E} 3$ & 1.88 & 2.65 & 0.54 & 2.00 & 0.03 & 3.42 & 0.03 & $\mathbf{1 . 2 0} \uparrow, \mathbf{1 . 3 4} \uparrow, 2.36 \uparrow, 2.43 \downarrow, 2.56 \downarrow, 2.95 \downarrow$ \\
\hline & $\mathrm{TH} \rightarrow \mathrm{TH}$ & 1.84 & 3.00 & 0.68 & 2.00 & -0.02 & 3.59 & 0.42 & $\mathbf{1 . 2 3} \uparrow, \mathbf{1 . 2 4} \uparrow, 1.93 \uparrow, 2.42 \downarrow, 2.45 \downarrow, 2.46 \downarrow$ \\
\hline \multirow[t]{2}{*}{ Ti } & $\mathrm{E} \rightarrow \mathrm{E} 2$ & 1.97 & 2.16 & 0.81 & 2.00 & 0.19 & 2.65 & 0.27 & $1.77 \uparrow, 2.30 \downarrow, 2.34 \uparrow, 2.43 \uparrow, 2.81 \uparrow, 2.89 \uparrow, 3.11 \uparrow$ \\
\hline & $\mathrm{TC} \rightarrow \mathrm{TC}$ & 1.95 & 2.52 & 0.89 & 3.91 & 0.31 & 2.16 & 0.92 & $\mathbf{2 . 1 8} \uparrow, \mathbf{2} .48 \uparrow, \mathbf{2 . 4 9} \uparrow, \mathbf{2 . 9 5} \uparrow, 3.18 \downarrow, 3.27 \uparrow, 3.28 \uparrow$ \\
\hline \multirow[t]{2}{*}{$\mathrm{V}$} & $\mathrm{E} \rightarrow \mathrm{E} 1$ & 1.99 & 2.40 & 0.54 & 5.00 & 0.14 & 2.56 & 0.22 & $1.49 \uparrow, 2.10 \uparrow, 2.38 \uparrow, 2.43 \uparrow, 2.50 \uparrow, 2.70 \uparrow, 3.16 \downarrow$ \\
\hline & $\mathrm{TC} \rightarrow \mathrm{M} 3$ & 1.71 & 2.07 & 1.24 & 3.00 & 0.93 & 3.13 & 0.43 & $\mathbf{0 . 1 0} \uparrow, \mathbf{0 . 4 9} \downarrow, \mathbf{1 . 4 5} \uparrow, \mathbf{1 . 6 0} \uparrow, \mathbf{1 . 8 4} \uparrow, 2.20 \uparrow, 3.03 \downarrow$ \\
\hline \multirow[t]{2}{*}{$\mathrm{Fe}$} & $\mathrm{E} \rightarrow \mathrm{E} 2$ & 1.90 & 2.15 & 0.57 & 4.00 & 0.05 & 3.21 & 0.36 & $-\mathbf{0} .42 \uparrow,-\mathbf{0} .27 \uparrow,-\mathbf{0 . 0 8} \uparrow,-\mathbf{0 . 0 3} \uparrow, \mathbf{0 . 0 3} \uparrow, 1.48 \downarrow, 1.59 \uparrow$ \\
\hline & $\mathrm{HO} \rightarrow \mathrm{HO}$ & 1.95 & 2.83 & 0.65 & 4.00 & 0.07 & 2.98 & 0.53 & $-\mathbf{1 . 1 5} \downarrow,-\mathbf{0} .75 \uparrow,-\mathbf{0} .57 \uparrow,-\mathbf{0} .41 \uparrow,-\mathbf{0} .34 \uparrow, \mathbf{0 . 0 9} \uparrow, 1.39 \uparrow$ \\
\hline \multirow[t]{2}{*}{$\mathrm{Ge}$} & $\mathrm{E} \rightarrow \mathrm{E} 3$ & 1.96 & 2.71 & 0.48 & 2.00 & 0.00 & 3.38 & 0.03 & $\mathbf{1 . 2 7} \uparrow, \mathbf{1 . 3 8} \uparrow, 2.25 \uparrow, 2.41 \downarrow, 2.52 \downarrow, 2.81 \downarrow$ \\
\hline & $\mathrm{TH} \rightarrow \mathrm{TH}$ & 1.91 & 3.06 & 0.62 & 2.00 & -0.02 & 3.55 & 0.36 & $\mathbf{1 . 3 0} \uparrow, \mathbf{1 . 3 1} \uparrow, 1.89 \uparrow, 2.38 \uparrow, 2.42 \uparrow, 2.43 \uparrow$ \\
\hline \multirow[t]{2}{*}{$\mathrm{Pt}$} & $\mathrm{E} \rightarrow \mathrm{E} 5$ & 1.56 & 2.00 & 3.79 & 0.00 & -0.13 & 4.57 & -0.09 & $-\mathbf{0 . 7 8},-\mathbf{0 . 3 6},-\mathbf{0 . 1 3}, 0.13,2.76$ \\
\hline & $\mathrm{TH} \rightarrow \mathrm{M} 2$ & 1.54 & 2.04 & 3.50 & 0.00 & -0.18 & 4.30 & 0.34 & $\mathbf{- 0 . 3 6},-\mathbf{0 . 2 1}, \mathbf{0 . 3 8}, 2.58,3.43$ \\
\hline
\end{tabular}

same figure the positions of adatoms after relaxation are also shown. Binding energies $\left(E_{b}\right)$ of these atoms are calculated by using the expression

$$
E_{b}=-E_{T}[\mathrm{NR}+A]+E_{T}[\mathrm{NR}]+E_{T}[A]
$$

in terms of the total energies of the optimized structure of graphane $\mathrm{NR}$ with adatom, $E_{T}[\mathrm{NR}+A]$; bare $\mathrm{NR}, E_{T}[\mathrm{NR}]$; and free adatom, $E_{T}[A]$. Owing to the large graphane nanoribbon unit cell, k-point sampling is done only along the axis of the ribbon. All the energies are calculated in the same unit cell and obtained from the lowest ground-state total energies (either magnetic or nonmagnetic). Initially, at least $2 \AA$ distance between adatom and outermost graphane atom is provided to find the relaxation site of adatom on the ribbon.

An atom adsorbed on graphane NR may give rise to resonance states in the valence and conduction bands, and also localized states in the band gap. Owing to the periodic boundary conditions, the energy levels associated with these adatoms may form energy bands. Since we consider a supercell consisting of six unit cell of $\mathrm{H}$-passivated armchair graphane NR, the adatom-adatom distance is large and prevents coupling between adjacent adatoms. In this respect, the flat bands associated with adatoms can mimic either resonance or localized states relevant for dilute doping.

In Table II, we present nearest-neighbor distances between adatom and $\mathrm{H}\left(d_{\mathrm{H}}\right)$ and $\mathrm{C}\left(d_{\mathrm{C}}\right)$, binding energy $\left(E_{b}\right)$, total magnetic moment of the system $\left(\mu_{T}\right)$, and charge transfer between adatom and adsorbate $\left(\rho^{*}\right)$. Since the inversion symmetry of graphane can be broken by the adsorption of an atom, a net electric dipole moment can be induced thereof. Thus electric dipole moment values calculated in the direction normal to graphane NR surface $p$, induced by adatom are also listed in Table II. Photoelectric threshold of the adatom+graphane system perpendicular to the plane of NR, $\Phi$, is calculated by the difference of the electrostatic potential at distances where the gradient of it is negligible and the Fermi level of the adsorbed system. It should be noted that both $p$ and $\Phi$ is relevant only for uniform coverage of graphane. For a dilute impurity, these calculated values converge to that of armchair graphane. When the width of armchair graphane NR is large enough, one expects the photoelectric threshold, $\Phi$, and dipole moment, $p$, to converge to $4.97 \mathrm{eV}$ and $0 \mathrm{e} \AA$, respectively. ${ }^{21}$

We found that some atoms, such as $\mathrm{C}, \mathrm{O}, \mathrm{V}$, and $\mathrm{Pt}$ that are adsorbed on the H-passivated armchair graphane NR surface show a tendency to remove $\mathrm{H}$ atoms from graphane surface. Since the single atoms of $\mathrm{C}, \mathrm{O}, \mathrm{V}$, and $\mathrm{Pt}$ have rather high binding energy, it seems possible to create graphene domains on graphane by striping $\mathrm{H}$ atoms but covering the domain by adsorbed atoms. Independent of their initial position, $\mathrm{C}$ and $\mathrm{O}$ atoms are adsorbed to carbon atoms of graphane by replacing $\mathrm{H}$ atoms. A typical binding configuration of $\mathrm{C}$ and $\mathrm{O}$ is indicated as M1 in Fig. 5. Adsorption of $\mathrm{C}$ and $\mathrm{O}$ atoms occur at the middle of nanoribbon with strong binding energies of $5.89 \mathrm{eV}$ and $3.99 \mathrm{eV}$, respectively. The resulting system is NM. Binding structures of $\mathrm{Pt}$ and $\mathrm{V}$, which also remove $\mathrm{H}$ atom from the host $\mathrm{C}$ atom of graphane, are also shown in the same figure. 

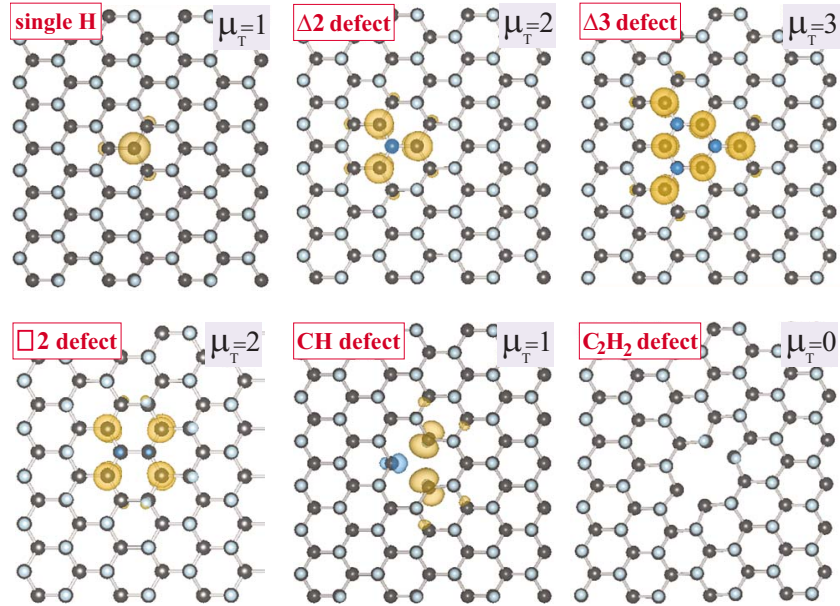
$-c \mu_{T}=$ - 0 . $0.0 \%$ - 0.00 000000 $00^{\circ} 00 \%$ 0 - 900 - 00 - 000

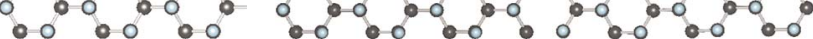

FIG. 6. (Color online) Atomic structures corresponding to single $\mathrm{H}$, double-sided triangular shaped $\Delta 2$ and $\Delta 3$, double-sided rectangular shaped $\square 2, \mathrm{CH}$ and $\mathrm{C}_{2} \mathrm{H}_{2}$ vacancies and resulting difference charge density, $\Delta \rho=\rho^{(\uparrow)}-\rho^{(\downarrow)}$, surfaces for a bare armchair graphane NR. Large/black and small/light blue-gray balls indicate $\mathrm{C}$ and $\mathrm{H}$ atoms, respectively. Only a small part which includes vacancy region and its nearby atoms, of the armchair graphane NR with $N$ $=15$ is shown.

Although $\mathrm{H}$ has very low binding energy (and hence is not shown in Table II), it may be important for striping $\mathrm{H}$ atoms from graphane. Upon all geometry relaxations from different initial conditions, single adsorbed $\mathrm{H}$ atom prefers to bind on top of $\mathrm{H}$ atom of graphane and forms $\mathrm{H}_{2}$ molecule which is weakly bound to the nearest $\mathrm{C}$ atom. The binding energy of $\mathrm{H}_{2}$ molecule is calculated to be $\sim 36 \mathrm{meV}$.

\section{VACANCIES IN GRAPHANE NRs}

In our recent study, ${ }^{21}$ we showed that a graphane, which has a NM ground state, can be made magnetic simply by removing hydrogen atoms from the uniform hydrogen layers adsorbed on its both sides. It was found that the magnetization depends on whether the defect region is one sided or two sided. It was also shown that in certain circumstances remarkably large magnetic moments can be attained in a small domain on the sheet of graphane. Experimentally, removal of surface hydrogen atoms by using laser beam resonating with surface-atom bond, ${ }^{34}$ ionic vapor application, ${ }^{35}$ and subnanometer Pt clusters ${ }^{36}$ are reported before. Since it is revealed that the double-sided vacancies give higher magnetic moments we focus on these type of vacancies on the nanoribbons.

In this section we consider six different types of vacancies in a bare armchair graphane NR with $N=15$. Upon desorption of a single hydrogen atom, as shown in Fig. 6, local bonding through $s p^{3}$-hybrid orbital is retransformed into planar $s p^{2}$ and perpendicular $p_{z}$ orbitals. At the vacancy site one electron accommodated by the dangling $p_{z}$ orbital becomes unpaired and hence contributes to the magnetization by $1 \mu_{B}$. We also calculated that a single $\mathrm{H}$ vacancy located at the center of the NR has $70 \mathrm{meV}$ lower energy than that located near the edge of the ribbon.
The situation is rather intriguing for the magnetization of large domains of hydrogen vacancies. As in the case of single $\mathrm{H}$ vacancy, leading to transformation of $s p^{3}$ bonding into planar $s p^{2}$ bonding, triangular $\Delta$, and rectangular $\square$ shaped large graphene islands can be created by removing $\mathrm{H}$ atoms hence by creating $\mathrm{H}$-vacancy domains. As shown in Fig. 6, hydrogen desorbed triangle-shaped island consisting of $n$ carbon atom at its each edge is defined as $\Delta n$-type $\mathrm{H}$ vacancy. Therefore, a $\Delta n$-type vacancy domain can be obtained by removing $n(n+1) / 2 \mathrm{H}$ atom from top side and $n(n-1) / 2 \mathrm{H}$ atom from down side of the graphane nanoribbon. This means that $n(n+1) / 2 p_{z}$-orbital electrons freed from A-type and $n(n-1) / 2 p_{z}$-orbital electrons freed from B-type sublattice $\mathrm{C}$ atoms. According to the Lieb's theorem ${ }^{37}$ net magnetic moment of such a system can be calculated from the difference between the sublattice atoms. For triangle vacancies this difference is simply equal to $n$ and thus the $\Delta n$-vacancy domain has net magnetic moment with $n \mu_{B}$. However, the $\square$-type domains do not yield large magnetic moments and have values between 0 and $2 \mu_{B}$. It is seen that magnetic interactions in double-sided triangular and square $\mathrm{H}$-vacancy domains resulting in net magnetic moments are relatively straightforward and are in good agreement with Lieb's theorem.

We also consider $\mathrm{CH}$ and $\mathrm{C}_{2} \mathrm{H}_{2}$ vacancies, the synthesis of them are relatively easier than creating only $\mathrm{H}$ vacancies. In our study, these vacancies are first created in a NR by removing involved atoms and subsequently their structures are optimized. Calculated values of magnetic moments of the structure are presented in Fig. 6. After the creation of a $\mathrm{CH}$ vacancy on a graphane nanoribbon, geometric structure rearranges itself via Jahn-Teller-type distortion in the lattice. Charge-density plot in Fig. 6 shows the unpaired up- and down-spin electron states located on the region surrounding the vacancy. It is seen that a bare armchair graphane NR, which is NM semiconductor when it is defect free, attains permanent magnetic moment in the presence of $\mathrm{CH}$ vacancies. While $\mathrm{CH}$-type vacancy makes small changes in the geometric structure, Stone-Wales-type deformation occurs after formation of the $\mathrm{C}_{2} \mathrm{H}_{2}$ vacancy. Since electrons are paired in $\mathrm{C}_{2} \mathrm{H}_{2}$ vacancy, the ground state is again $\mathrm{NM}$ and thus there is no visible difference charge density in Fig. 6. From the point view of Lieb's theorem, removal of a $\mathrm{CH}$ couple results in an absence in the number of $p_{z}$-orbital electron belonging to a A-type (or B-type) sublattice. Upon the creation of $\mathrm{C}_{2} \mathrm{H}_{2}$ vacancy, both the A- and B-type sublattices lose one electron and thus the difference is zero resulting in a NM ground state. Our results are consistent with the recently reported values for $\mathrm{CH}$ vacancies in infinite sheets of graphane. $^{38}$

\section{EDGE ROUGHNESS}

Earlier, it was shown that the edge roughness of graphene NRs can affect their electronic ${ }^{39-41}$ and mechanical ${ }^{42}$ properties. In particular, it was revealed that periodically repeating edge profiles can be treated within the superlattice structure, which result in confinement of spin states in zigzag NRs. ${ }^{43}$ Band alignments of these superlattice structures have been 

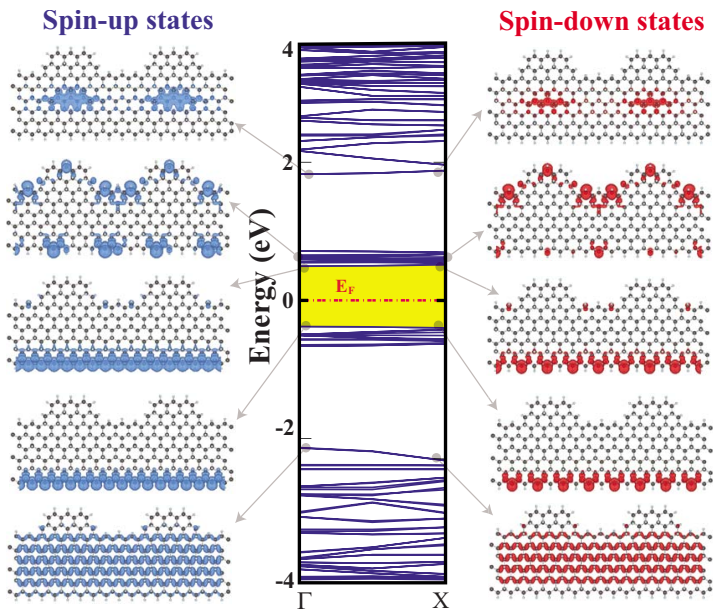

FIG. 7. (Color online) Energy-band diagram and band projected charge-density isosurfaces of various states for bare zigzag graphane NR including edge roughness. The band gap between edge states are shaded yellow/gray. Zero of the band energy is set to the Fermi level.

studied both experimentally and theoretically. ${ }^{8}$ Here we investigated the effect of periodically repeating edge roughness of a bare zigzag graphane NR. While one edge of NR is kept flat, periodically repeating undulations are carved at the other edge. The same structure can be viewed as periodically repeating heterostructures of wide $(N=8)$ and narrow $(N=6)$ segments of zigzag graphane NRs. Atomic and electronic band structure of this superlattice, which mimics the edge roughness are shown in Fig. 7. Here we note that infinite bare zigzag graphane NR with $N=8$ has a band gap of $0.8 \mathrm{eV}$ while the band gap of infinite bare zigzag graphane NR with $N=6$ is relatively wider and $0.9 \mathrm{eV}$. As pointed out in Sec. $\mathrm{IV}$, wide band gap of $\mathrm{H}$-passivated graphane NRs is reduced to $0.8-0.9 \mathrm{eV}$ because of edge states of unpassivated dangling bonds of bare graphane NRs, which appear in the band gap. These edge states in Fig. 7 of the superlattice occur as several closely lying flat bands below and above the Fermi level separated by a superlattice band gap of $\sim 0.8 \mathrm{eV}$. The character of various bands are revealed by plotting the charge-density isosurfaces of various states. Charge density isosurfaces of the highest filled spin-up and spin-down edge state indicate the localization at the flat edge. As for the charge distribution of the lower lying valence states is rather uniform along the narrow region. The states of the flat band at the edge of the conduction band is confined in the wide segment of the superlattice since they cannot find a matching state in the narrow region. As a proof of the concept, it is shown that electronic states can be confined at specific regions of periodic edge roughness. Of course, confinement, superlattice band gap, etc., depend on the structural parameters of superlattices and need detailed investigation.

\section{DISCUSSIONS AND CONCLUSIONS}

Research on recently synthesized graphane revealed interesting electronic and magnetic properties of this twodimensional honeycomb structure. Graphane attains a $s p^{3}$-like bonding through covering of its both sides by hydrogen atom. This bonding is rather different from the $s p^{2}$ bonding of graphene and attributes a number of additional properties to graphane. For example, in contrast to semimetallic graphene with linear band crossing at the Fermi level, graphane is a nonmagnetic, wideband semiconductor.

The armchair and zigzag graphane NRs of graphane display important number of properties and hence constitute basic structures to fabricate various devices. Both zigzag and armchair graphane NRs are wide band-gap semiconductor when their edges are passivated with hydrogen. The band gaps vary exponentially with their widths. For narrow graphane NRs the band gap is large due to quantum confinement effect but approaches to the band gap of graphane as the width increases. Unpaired, unpassivated dangling bonds at the edges have $1 \mu_{B}$ magnetic moment and have antiferromagnetic coupling with adjacent dangling bonds. These graphane NRs can be functionalized to attain additional properties through $\mathrm{H}$ passivation of their edges, adatom adsorption, vacancy creation, edge profiling, and superlattice formation. In particular, graphane NRs attain magnetic moment through the creation of $\mathrm{H}$ vacancy at the surfaces of graphane. This property can be utilized to achieve interesting functionalities through decoration and patterning of $\mathrm{H}$ vacancies on the graphane NRs. The possibility of generation of large magnetic moments at small domains of $\mathrm{H}$ vacancies makes graphane based structures promising for data storage and nanospintronic applications. These functionalities can be further extended by adsorbing adatoms to carbon atoms deprived from hydrogen. In conclusion, the present study demonstrates that graphane NRs can be an important basic nanomaterial and presents interesting properties for future technological applications.

\section{ACKNOWLEDGMENTS}

Computing resources used in this work were partly provided by the National Center for High Performance Computing of Turkey (UYBHM) under Grant No. 2-024-2007. This work is partially supported by the project of The State Planning Organization (DPT) of Turkey and by Academy of Science of Turkey (TÜBA).

\footnotetext{
*ciraci@fen.bilkent.edu.tr

${ }^{1}$ K. S. Novoselov, D. Jiang, F. Schedin, T. J. Booth, V. V. Khotkevich, S. V. Morozov, and A. K. Geim, Science 306, 666 (2004).
}

\footnotetext{
${ }^{2}$ K. S. Novoselov, A. K. Geim, S. V. Morozov, D. Jiang, M. I Katsnelson, I. V. Grigorieva, S. V. Dubonos, and A. A. Firsov, Nature (London) 438, 197 (2005).

${ }^{3}$ Y. Zhang, Y.-W. Tan, H. L. Stormer, and Philip Kim, Nature
} 
(London) 438, 201 (2005).

${ }^{4}$ C. Berger, Z. Song, X. Li, X. Wu, N. Brown, C. Naud, D. Mayou, T. Li, J. Hass, A. N. Marchenkov, E. H. Conrad, P. N. First, and W. A. de Heer, Science 312, 1191 (2006).

${ }^{5}$ M. I. Katsnelson, K. S. Novoselov, and A. K. Geim, Nat. Phys. 2, 620 (2006).

${ }^{6}$ A. K. Geim and K. S. Novoselov, Nature Mater. 6, 183 (2007).

${ }^{7}$ M. Y. Han, B. Özyilmaz, Y. Zhang, and P. Kim, Phys. Rev. Lett. 98, 206805 (2007).

${ }^{8}$ X. Li, X. Wang, L. Zhang, S. Lee, and H. Dai, Science 319, 1229 (2008).

${ }^{9}$ H. Lee, Y. W. Son, N. Park, S. Han, and J. Yu, Phys. Rev. B 72, 174431 (2005).

${ }^{10}$ V. Barone, O. Hod, and G. E. Scuseria, Nano Lett. 6, 2748 (2006).

${ }^{11}$ D. A. Abanin, P. A. Lee, and L. S. Levitov, Phys. Rev. Lett. 96, 176803 (2006).

${ }^{12}$ A. Cresti, G. Grosso, and G. P. Parravicini, Phys. Rev. B 76, 205433 (2007).

${ }^{13}$ M. Ezawa, Phys. Rev. B 73, 045432 (2006).

${ }^{14}$ J. Guo, D. Gunlycke, and C. T. White, Appl. Phys. Lett. 92, 163109 (2008).

${ }^{15}$ O. Hod, V. Barone, J. E. Peralta, and G. E. Scuseria, Nano Lett. 7, 2295 (2007).

${ }^{16}$ D. C. Elias, R. R. Nair, T. M. G. Mohiuddin, S. V. Morozov, P. Blake, M. P. Halsall, A. C. Ferrari, D. W. Boukhvalov, M. I. Katsnelson, A. K. Geim, and K. S. Novoselov, Science 323, 610 (2009).

${ }^{17}$ J. O. Sofo, A. S. Chaudhari, and G. D. Barber, Phys. Rev. B 75, 153401 (2007).

${ }^{18}$ D. W. Boukhvalov, M. I. Katsnelson, and A. I. Lichtenstein, Phys. Rev. B 77, 035427 (2008).

${ }^{19}$ M. Z. S. Flores, P. A. S. Autreto, S. B. Legoas, and D. S. Galvao, Nanotechnology 20, 465704 (2009).

${ }^{20}$ Y. Li, Z. Zhou, P. Shen, and Z. Chen, J. Phys. Chem. C 113, 15043 (2009).

${ }^{21}$ H. Şahin, C. Ataca, and S. Ciraci, Appl. Phys. Lett. 95, 222510 (2009).
${ }^{22}$ S. Lebègue, M. Klintenberg, O. Eriksson, and M. I. Katsnelson, Phys. Rev. B 79, 245117 (2009).

${ }^{23}$ G. Kresse and J. Hafner, Phys. Rev. B 47, 558 (1993); G. Kresse and J. Furthmüller, ibid. 54, 11169 (1996).

${ }^{24}$ P. E. Blöchl, Phys. Rev. B 50, 17953 (1994).

${ }^{25}$ D. M. Ceperley and B. J. Alder, Phys. Rev. Lett. 45, 566 (1980).

${ }^{26}$ M. Shishkin and G. Kresse, Phys. Rev. B 74, 035101 (2006).

${ }^{27}$ M. R. Sorensen, M. Brandbyge, and K. W. Jacobsen, Phys. Rev. B 57, 3283 (1998).

${ }^{28}$ H. Şahin, S. Cahangirov, M. Topsakal, E. Bekaroglu, E. Akturk, R. T. Senger, and S. Ciraci, Phys. Rev. B 80, 155453 (2009).

${ }^{29}$ C. Lee, X. Wei, J. W. Kysar, and J. Hone, Science 321, 385 (2008).

${ }^{30}$ M. Topsakal, S. Cahangirov, and S. Ciraci, Appl. Phys. Lett. 96, 091912 (2010).

${ }^{31}$ H. Şahin and R. T. Senger, Phys. Rev. B 78, 205423 (2008).

${ }^{32}$ C. Ataca, E. Aktürk, and S. Ciraci, Phys. Rev. B 79, 041406(R) (2009).

${ }^{33}$ K. T. Chan, J. B. Neaton, and M. L. Cohen, Phys. Rev. B 77, 235430 (2008).

${ }^{34}$ Z. Liu, L. C. Feldman, N. H. Tolk, Z. Zhang, and P. I. Cohen, Science 312, 1024 (2006).

${ }^{35}$ L. Breaux, B. Anthony, T. Hsu, S. Banerjee, and A. Tasch, Appl. Phys. Lett. 55, 1885 (1989).

${ }^{36}$ S. Vajda, M. J. Pellin, J. P. Greeley, C. L. Marshall, L. A. Curtiss, G. A. Ballentine, J. W. Elam, S. Catillon-Mucherie, P. C. Redfern, F. Mehmood, and P. Zapol, Nature Mater. 8, 213 (2009).

${ }^{37}$ E. H. Lieb, Phys. Rev. Lett. 62, 1201 (1989).

${ }^{38}$ B. S. Pujari and D. G. Kanhere, J. Phys. Chem. C 113, 21063 (2009).

${ }^{39}$ D. Gunlycke, D. A. Areshkin, and C. T. White, Appl. Phys. Lett. 90, 142104 (2007).

${ }^{40}$ Y. Yoon and J. Guo, Appl. Phys. Lett. 91, 073103 (2007).

${ }^{41}$ M. Evaldsson, I. V. Zozoulenko, H. Xu, and T. Heinzel, Phys. Rev. B 78, 161407(R) (2008).

${ }^{42}$ H. Şahin, C. Ataca, and S. Ciraci (unpublished).

${ }^{43}$ H. Sevinçli, M. Topsakal, and S. Ciraci, Phys. Rev. B 78, 245402 (2008). 